\title{
Article
}

\section{Strong convergence theorems of common fixed points for a uniformly closed asymptotically family of countable quasi-Lipschitz mappings in Hilbert spaces}

\author{
Afshan Perveen ${ }^{1}$, Samina Kausar ${ }^{2}$ and Waqas Nazeer ${ }^{2, *}$ \\ 1 Department of Mathematics, The University of Lahore Pakpattan Campus, Pakistan.; mmzia99@gmail.com \\ 2 Division of Science and Technology, University of Education, Lahore, Pakistan.; sminasaddique@gmail.com (S.K) \\ * Correspondence: nazeer.waqas@ue.edu.pk
}

Received: 15 September 2018; Accepted: 7 November 2018; Published: 21 February 2019.

\begin{abstract}
In this paper, we present a new non-convex hybrid iteration algorithm for common fixed points of a uniformly closed asymptotically family of countable quasi-Lipschitz mappings in the domains of Hilbert spaces.
\end{abstract}

Keywords: S iteration, nonexpansive mapping, hybrid algorithm, closed quasi-nonexpansive.

MSC: 47H05, 47H09, 47H10

\section{Introduction}

$\mathbf{F}$ ixed point theory of special mappings like nonexpansive, asymptotically nonexpansive, contractive and other mappings is an active area of interest and finds applications in many related fields like image recovery, signal processing and geometry of objects [1]. From time to time, some versions of theorems relating to fixed points of functions of special nature keep on appearing in almost in all branches of mathematics. Consequently, we apply them in industry, toy making, finance, aircrafts and manufacturing of new model cars. For example, a fixed-point iteration scheme has been applied in IMRT optimization to pre-compute dose-deposition coefficient (DDC) matrix, see [2]. Because of its vast range of applications almost in all directions, the research in it is moving rapidly and an immense literature is currently.

The construction of fixed point theorems (e.g. Banach fixed point theorem) which not only claim the existence of a fixed point but yield an algorithm, too (in the Banach case fixed point iteration $x_{n+1}=f\left(x_{n}\right)$ ). Any equation that can be written as $x=f(x)$ for some map $f$ that is contracting with respect to some (complete) metric on $X$ will provide such a fixed point iteration. Mann's iteration method was the stepping stone in this regard and is invariably used in most of the occasions, see [3]. But it only ensures weak convergence, see [4] but more often then not, we require strong convergence in many real world problems relating to Hilbert spaces, see [5]. So mathematician are in search for the modifications of the Mann's process to control and ensure the strong convergence, (see [4,6-12] and references therein).

Most probably the first noticeable modification of Mann's iteration process was proposed by Nakajo et al. in [8] in 2003. They introduced this modification for only one nonexpansive mapping, where as Kim and $\mathrm{Xu}$ introduced a modification for asymptotically nonexpansive mapping in 2006, see [9]. In the same year Martinez et al. in [10] introduced a modification of the Ishikawa iteration process for a nonexpansive mapping. They also gave modification of Halpern iteration method. Su et al. in [11] gave a monotone hybrid iteration process for nonexpansive mapping. Liu et al. in [12] gave a novel iteration method for finite family of quasi-asymptotically pseudo-contractive mapping in a Hilbert space. Let $H$ be the fixed notation for Hilbert space and $C$ be nonempty, closed and convex subset of it. First we recall some basic definitions that will accompany us throughout this paper.

Let $P_{C}($.$) be the metric projection onto C$. A mapping $T: C \rightarrow C$ is said to be non-expensive if $\|T x-T y\| \leq$ $\|x-y\| \forall x, y \in C$. And $T: C \rightarrow C$ is said to be quasi-Lipschitz if

1. $\operatorname{Fix} T \neq \phi$, 
2. For all $p \in$ FixT, $\|T x-p\| \leq L\|x-p\|$ where $L$ is a constant $1 \leq L<\infty$.

If $L=1$ then $T$ is known as quasi-nonexpansive. It is well-known that $T$ is said to be closed if for $n \rightarrow \infty$, $x_{n} \rightarrow x$ and $\left\|T x_{n}-x_{n}\right\| \rightarrow 0$ implies $T x=x$. $T$ is said to be weak closed if $x_{n} \rightarrow x$ and $\left\|T x_{n}-x_{n}\right\| \rightarrow 0$ implies $T x=x$. as $n \rightarrow \infty$. It is admitted fact that a mapping which is weak closed should be closed but converse is no longer true.

Let $\left\{T_{n}\right\}$ be a sequence of mappings having non-empty fixed points sets. Then $\left\{T_{n}\right\}$ is defined to be uniformly closed if for all convergent sequences $\left\{Z_{n}\right\} \subset C$ with conditions $\left\|Z x_{n}-Z_{n}\right\| \rightarrow 0, n \rightarrow \infty$ implies the limit of $\left\{Z_{n}\right\}$ belongs to FixT $T_{i}$.

In 1953 [3], Mann proposed an iterative scheme given as:

$$
x_{n+1}=\left(1-\alpha_{n}\right) x_{n} n+\alpha_{n} T\left(x_{n}\right) ; n=0,1,2, \ldots
$$

Guan et al. in [7] established the following non-convex hybrid iteration algorithm corresponding to Mann iterative scheme:

$$
\begin{cases}x_{0} \in C=Q_{0}, & \text { choosen arbitrarily, } \\ y_{n}=\left(1-\alpha_{n}\right) x_{n}+\alpha_{n} T_{n} x_{n}, & n \geq 0 \\ C_{n}=\left\{z \in C:\left\|y_{n}-z\right\| \leq\left(1+\left(L_{n}-1\right) \alpha_{n}\right)\left\|x_{n}-z\right\| \cap A,\right. & n \geq 0 \\ Q_{n}=\left\{z \in Q_{n-1}:\left\langle x_{n}-z, x_{0}-x_{n}\right\rangle \geq 0\right\}, & n \geq 1 \\ x_{n+1}=P_{\overline{c o} C_{n} \cap Q_{n}} x_{0} & \end{cases}
$$

They proved strong convergence results relating to common fixed points for a uniformly closed asymptotic family of countable quasi-Lipschitz mappings in $H$. They applied their results for the finite case to obtain fixed points. In this article, we establish a non-convex hybrid algorithm and prove strong convergence theorems about common fixed points related to a uniformly closed asymptotically family of countable quasi-Lipschitz mappings in the realm of Hilbert spaces. An application of this algorithm is also given.

We fix $\overline{c o} C_{n}$ for closed convex closure of $C_{n}$ for all $n \geq 1, A=\left\{z \in H:\left\|z-P_{F} x_{0}\right\| \leq 1\right\}, T_{n}$ for countable quasi- $L_{n}$-Lipschitz mappings from $C$ into itself, and $T$ be closed quasi-nonexpansive mapping from $C$ into itself to avoid redundancy. We also present an application of our algorithm.

\section{Main results}

In this part we formulate our main results. We start with some basic definitions.

Definition 1. $\left\{T_{n}\right\}$ is said to be asymptotic, if $\lim _{n \rightarrow \infty} L_{n}=1$

Proposition 2. For $x \in H$ and $z \in C, z=P_{C} x$ iff we have $\langle x-z, z-y\rangle \geq 0$ for all $y \in C$.

Proposition 3. The common fixed point set $F$ of above said $T_{n}$ is closed and convex.

Proposition 4. For any given $x_{0} \in H$, we have $p=P_{C} x_{0} \Longleftrightarrow\left\langle p-z, x_{0}-p\right\rangle \geq 0, \forall z \in C$.

Theorem 5. Suppose that $\alpha_{n} \in(0,1]$, and $\beta_{n} \in[0,1]$ for all $n \in N$. Then $\left\{x_{n}\right\}$ generated by

$$
\begin{cases}x_{0} \in C=Q_{0}, & \text { choosen arbitrarily, } \\ y_{n}=\left(1-\alpha_{n}\right) T_{n} x_{n}+\alpha_{n} T_{n} z_{n}, & n \geq 0 \\ z_{n}=\left(1-\beta_{n}\right)+\beta_{n} T_{n} x_{n}, & n \geq 0 \\ C_{n}=\left\{z \in C:\left\|y_{n}-z\right\| \leq L_{n}\left(1+\left(L_{n}-1\right) \alpha_{n} \beta_{n}\right)\left\|x_{n}-z\right\|\right\} \cap A, & n \geq 0 \\ Q_{n}=\left\{z \in Q_{n-1}:\left\langle x_{n}-z, x_{0}-x_{n}\right\rangle \geq 0\right\}, & n \geq 1 \\ x_{n+1}=P_{\overline{c o} C_{n} \cap Q_{n}} x_{0}, & \end{cases}
$$

converges strongly to $P_{F} x_{0}$. 
Proof. We give our proof in following steps.

Step 1. We know that $\overline{c o} C_{n}$ and $Q_{n}$ are closed and convex for all $n \geq 0$. Next, we show that $F \cap A \subset \overline{c o} C_{n}$ for all $n \geq 0$. Indeed, for each $p \in F \cap A$, we have

$$
\begin{aligned}
\left\|y_{n}-p\right\| & =\left\|\left(1-\alpha_{n}\right) T_{n} x_{n}+\alpha_{n} T_{n} z_{n}-p\right\| \\
& =\left\|\left(1-\alpha_{n}\right) T_{n} x_{n}+\alpha_{n} T_{n}\left(\left(1-\beta_{n}\right)+\beta_{n} T_{n} x_{n}\right)-p\right\| \\
& =\left\|\left(1-\alpha_{n} \beta_{n}\right)\left(T_{n} x_{n}-p\right)+\left(\alpha_{n} \beta_{n}\right)\left(T_{n}^{2} x_{n}\right)\right\| \\
& \leq\left(1-\alpha_{n} \beta_{n}\right)\left\|T_{n} x_{n}-p\right\|+\left(\alpha_{n} \beta_{n}\right)\left\|T_{n}^{2} x_{n}\right\| \\
& =L_{n}\left(1+\left(L_{n}-1\right) \alpha_{n} \beta_{n}\right)\left\|x_{n}-p\right\|
\end{aligned}
$$

and $p \in A$, so $p \in C_{n}$ which implies that $F \cap A \subset C_{n}$ for all $n \geq 0$. therefore, $F \cap A \subset \overline{c o} C_{n}$ for all $n \geq 0$.

Step 2. We show that $F \cap A \subset \overline{c o} C_{n} \cap Q_{n}$ for all $n \geq 0$. it suffices to show that $F \cap A \subset Q_{n}$, for all $n \geq 0$. We prove this by mathematical induction. For $n=0$ we have $F \cap A \subset C=Q_{0}$. Assume that $F \cap A \subset Q_{n}$. Since $x_{n+1}$ is the projection of $x_{0}$ onto $\overline{c o} C_{n} \cap Q_{n}$, from Proposition 3, we have

$\left\langle x_{n+1}-z, x_{n+1}-x_{0}\right\rangle \leq 0, \forall z \in \overline{c o} C_{n} \cap Q_{n}$

as $F \cap A \subset \overline{c o} C_{n} \cap Q_{n}$, the last inequality holds, in particular, for all $z \in F \cap A$. This together with the definition of $Q_{n+1}$ implies that $F \cap A \subset Q_{n+1}$. Hence the $F \cap A \subset \overline{c o} C_{n} \cap Q_{n}$ holds for all $n \geq 0$.

Step 3. We prove $\left\{x_{n}\right\}$ is bounded. Since $F$ is a nonempty, closed, and convex subset of $C$, there exists a unique element $z_{0} \in F$ such that $z_{0}=P_{F} x_{0}$. From $x_{n+1}=P_{\overline{c o} C_{n} \cap Q_{n}} x_{0}$, we have

$\left\|x_{n+1}-x_{0}\right\| \leq\left\|z-x_{0}\right\|$

for every $z \in \overline{c o} C_{n} \cap Q_{n}$. As $z_{0} \in F \cap A \subset \overline{c o} C_{n} \cap Q_{n}$, we get

$\left\|x_{n+1}-x_{0}\right\| \leq\left\|z_{0}-x_{0}\right\|$

for each $n \geq 0$. This implies that $\left\{x_{n}\right\}$ is bounded.

Step 4. We show that $\left\{x_{n}\right\}$ converges strongly to a point of $C$ (we show that $\left\{x_{n}\right\}$ is a cauchy sequence). As $x_{n+1}=P_{\overline{c o} C_{n} \cap Q_{n}} x_{0} \subset Q_{n}$ and $x_{n}=P_{Q_{n}} x_{0}$ (Proposition 4), we have

$\left\|x_{n+1}-x_{0}\right\| \geq\left\|x_{n}-x_{0}\right\|$

for every $n \geq 0$, which together with the boundedness of $\left\|x_{n}-x_{0}\right\|$ implies that there exists the limit of $\left\|x_{n}-x_{0}\right\|$. On the other hand, from $x_{n+m} \in Q_{n}$, we have $\left\langle x_{n}-x_{n+m}, x_{n}-x_{0}\right\rangle \leq 0$ and hence

$$
\begin{aligned}
\left\|x_{n+m}-x_{n}\right\|^{2} & =\left\|\left(x_{n+m}-x_{0}\right)-\left(x_{n}-x_{0}\right)\right\|^{2} \\
& \leq\left\|x_{n+m}-x_{0}\right\|^{2}-\left\|x_{n}-x_{0}\right\|^{2}-2\left\langle x_{n+m}-x_{n}, x_{n}-x_{0}\right\rangle \\
& \leq\left\|x_{n+m}-x_{0}\right\|^{2}-\left\|x_{n}-x_{0}\right\|^{2} \rightarrow 0 \text { as } n \rightarrow \infty
\end{aligned}
$$

for any $m \geq 1$. Therefore $\left\{x_{n}\right\}$ is a cauchy sequence in $C$, then there exists a point $q \in C$ such that $\lim _{n \rightarrow \infty} x_{n}=$ $q$.

Step 5 . We show that $y_{n} \rightarrow q$, as $n \rightarrow \infty$. Let

$D_{n}=\left\{z \in C:\left\|y_{n}-z\right\|^{2} \leq\left\|x_{n}-z\right\|^{2}+L_{n}^{2}\left(L_{n}-1\right)\left(L_{n}+1\right)\right\}$.

From the definition of $D_{n}$, we have

$$
\begin{aligned}
D_{n} & =\left\{z \in C:\left\langle y_{n}-z, y_{n}-z\right\rangle \leq\left\langle x_{n}-z, x_{n}-z\right\rangle+L_{n}^{2}\left(L_{n}-1\right)\left(L_{n}+1\right)\right\} \\
& =\left\{z \in C:\left\|y_{n}\right\|^{2}-2\left\langle y_{n}, z\right\rangle+\|z\|^{2} \leq\left\|x_{n}\right\|^{2}-2\left\langle x_{n}, z\right\rangle+\|z\|^{2}+L_{n}^{2}\left(L_{n}-1\right)\left(L_{n}+1\right)\right\} \\
& =\left\{z \in C: 2\left\langle x_{n}-y_{n}, z\right\rangle \leq\left\|x_{n}\right\|^{2}-\left\|y_{n}\right\|^{2}+L_{n}^{2}\left(L_{n}-1\right)\left(L_{n}+1\right)\right\}
\end{aligned}
$$

This shows that $D_{n}$ is convex and closed, $n \in \mathbb{Z}^{+} \cup\{0\}$. Next, we want to prove that $C_{n} \subset D_{n}, n \geq 0$. In fact, for any $z \in C_{n}$, we have

$$
\begin{aligned}
\left\|y_{n}-z\right\|^{2} & \leq\left[L_{n}\left(1+\left(L_{n}-1\right) \alpha_{n} \beta_{n}\right)\right]^{2}\left\|x_{n}-z\right\|^{2} \\
& =\left\|x_{n}-z\right\|^{2} L_{n}^{2}+L_{n}^{2}\left[2\left(L_{n}-1\right) \alpha_{n} \beta_{n}+\left(L_{n}-1\right)^{2} \alpha_{n}^{2} \beta_{n}^{2}\right]\left\|x_{n}-z\right\|^{2} \\
& \leq\left\|x_{n}-z\right\|^{2} L_{n}^{2}+L_{n}^{2}\left[2\left(L_{n}-1\right)+\left(L_{n}-1\right)^{2}\right]\left\|x_{n}-z\right\|^{2} \\
& =\left\|x_{n}-z\right\|^{2} L_{n}^{2}+L_{n}^{2}\left(L_{n}-1\right)\left(L_{n}+1\right)\left\|x_{n}-z\right\|^{2} .
\end{aligned}
$$


From $C_{n}=\left\{z \in C:\left\|y_{n}-z\right\| \leq\left[L_{n}\left(1+\left(L_{n}-1\right) \alpha_{n} \beta_{n}\right)\right]\left\|x_{n}-z\right\|\right\} \cap A, n \geq 0$, we have $C_{n} \subset A, n \geq 0$. Since $A$ is convex, we also have $\overline{c o} C_{n} \subset A, n \geq 0$. Consider $x_{n} \in \overline{c o} C_{n-1}$, we know that

$$
\begin{aligned}
\left\|y_{n}-z\right\| & \leq\left\|x_{n}-z\right\|^{2} L_{n}^{2}+L_{n}^{2}\left(L_{n}-1\right)\left(L_{n}+1\right)\left\|x_{n}-z\right\|^{2} \\
& \leq\left\|x_{n}-z\right\|^{2}+L_{n}^{2}\left(l_{n}-1\right)\left(L_{n}+1\right) .
\end{aligned}
$$

This implies that $z \in D_{n}$ and hence $C_{n} \subset D_{n}, n \geq 0$. Sinnce $D_{n}$ is convex, we have $\overline{c o}\left(C_{n}\right) \subset D_{n}, n \geq 0$. Therefore $\left\|y_{n}-x_{n+1}\right\|^{2} \leq\left\|x_{n}-x_{n+1}\right\|^{2}+L_{n}^{2}\left(L_{n}-1\right)\left(L_{n}-1\right) \rightarrow 0$ as $n \rightarrow \infty$. That is, $y_{n} \rightarrow q$ as $n \rightarrow \infty$.

Step 6. We show that $q \in F$. From the definition of $y_{n}$, we have $\left(1+\alpha_{n} \beta_{n} T_{n}\right)\left\|T_{n} x_{n}-x_{n}\right\|=\left\|y_{n}-x_{n}\right\| \rightarrow 0$ as $n \rightarrow \infty$. Since $\alpha_{n} \in(a, 1] \subset[0,1]$,from the above limit we have $\lim _{n} \rightarrow \infty\left\|T_{n} x_{n}-x_{n}\right\|=0$.

Since $\left\{T_{n}\right\}$ is uniformly closed and $x_{n} \rightarrow q$, we have $q \in F$.

Step 7. We claim that $q=z_{0}=P_{F} x_{0}$, if not, we have that $\left\|x_{0}-p\right\|>\left\|x_{0}-z_{0}\right\|$. There must exist a positive integer $N$, if $n>N$ then $\left\|x_{0}-x_{n}\right\|>\left\|x_{0}-z_{0}\right\|$, which leads to

$\left\|z_{0}-x_{n}\right\|^{2}=\left\|z_{0}-x_{n}+x_{n}-x_{0}\right\|^{2}=\left\|z_{0}-x_{n}\right\|^{2}+\left\|x_{n}-x_{0}\right\|^{2}+2\left\langle z_{0}-x_{n}, x_{n}-x_{0}\right\rangle$.

It follows that $\left\langle z_{0}-x_{n}, x_{n}-x_{0}\right\rangle<0$ which implies that $z_{0} \bar{\in} Q_{n}$, so that $z_{0} \bar{\in} F$, this is a contradiction. This completes the proof.

Now, we present an example of $C_{n}$ which does not involve a convex subset.

Example 1. Take $H=R^{2}$, and a sequence of mappings $T_{n}: R^{2} \rightarrow R^{2}$ given by $T_{n}:\left(t_{1}, t_{2}\right) \mapsto\left(\frac{1}{8} t_{1}, t_{2}\right)$, $\forall\left(t_{1}, t_{2}\right) \in R^{2}, \forall n \geq 0$.

It is clear that $\left\{T_{n}\right\}$ satisfies the desired definition of with $F=\left\{\left(t_{1}, 0\right): t_{1} \in(-\infty,+\infty)\right\}$ common fixed point set. Take $x_{0}=(4,0), a_{0}=\frac{6}{7}$, we have

$y_{0}=\frac{1}{7} x_{0}+\frac{6}{7} T_{0} x_{0}=\left(4 \times \frac{1}{7}+\frac{4}{8} \times \frac{6}{7}, 0\right)=(1,0)$.

Take $1+\left(L_{0}-1\right) a_{0}=\sqrt{\frac{5}{2}}$, we have

$C_{0}=\left\{z \in R^{2}:\left\|y_{0}-z\right\| \leq \sqrt{\frac{5}{2}}\left\|x_{0}-z\right\|\right\}$.

It is easy to show that $z_{1}=(1,3), z_{2}=(-1,3) \in C_{0}$. But

$z^{\prime}=\frac{1}{2} z_{1}+\frac{1}{2} z_{2}=(0,3) \bar{\epsilon} C_{0}$,

since $\left\|y_{0}-z\right\|=2,\left\|x_{0}-z\right\|=1$. Therefore $C_{0}$ is not convex.

Corollary 6. Assume that $\alpha_{n} \in(0,1]$, and $\beta_{n} \in[0,1]$ for all $n \in N$. Then $\left\{x_{n}\right\}$ generated by

$$
\begin{cases}x_{0} \in C=Q_{0}, & \text { choosen arbitrarily, } \\ y_{n}=\left(1-\alpha_{n}\right) T x_{n}+\alpha_{n} T z_{n}, & n \geq 0 \\ z_{n}=\left(1-\beta_{n}\right)+\beta_{n} T x_{n}, & n \geq 0 \\ C_{n}=\left\{z \in C:\left\|y_{n}-z\right\| \leq\left\|x_{n}-z\right\|\right\} \cap A, & n \geq 0 \\ Q_{n}=\left\{z \in Q_{n-1}:\left\langle x_{n}-z, x_{0}-x_{n}\right\rangle \geq 0\right\}, & n \geq 1 \\ x_{n+1}=P_{C_{n} \cap Q_{n}} x_{0}, & \end{cases}
$$

converges strongly to $P_{F(T)} x_{0}$.

Proof. Take $T_{n} \equiv T, L_{n} \equiv 1$ in Theorem 5 , in this case, $C_{n}$ is convex and closed and, for all $n \geq 0$, by using Theorem1.9, we obtain our desired result.

Corollary 7. Assume that $\alpha_{n} \in(0,1]$, and $\beta_{n} \in[0,1]$ for all $n \in N$. Then $\left\{x_{n}\right\}$ generated by

$$
\begin{cases}x_{0} \in C=Q_{0}, & \text { choosen arbitrarily, } \\ y_{n}=\left(1-\alpha_{n}\right) T x_{n}+\alpha_{n} T z_{n}, & n \geq 0 \\ z_{n}=\left(1-\beta_{n}\right)+\beta_{n} T x_{n}, & n \geq 0 \\ C_{n}=\left\{z \in C:\left\|y_{n}-z\right\| \leq\left\|x_{n}-z\right\|\right\} \cap A, & n \geq 0 \\ Q_{n}=\left\{z \in Q_{n-1}:\left\langle x_{n}-z, x_{0}-x_{n}\right\rangle \geq 0\right\}, & n \geq 1 \\ x_{n+1}=P_{C_{n} \cap Q_{n}} x_{0}, & \end{cases}
$$

converges strongly to $P_{F(T)} x_{0}$. 


\section{Application}

Here, we give an application of our result for the following case of finite family of asymptotically quasi-nonexpansive mappings $\left\{T_{n}\right\}_{n=0}^{N-1}$. Let

$\left\|T_{i}^{j} x-p\right\| \leq k_{i, j}\|x-p\|, \forall x \in C, p \in F$,

where $F$ is common fixed point set of $\left\{T_{n}\right\}_{n=0}^{N-1}, \lim _{j} \rightarrow \infty k_{i, j}=1$ for all $0 \leq i \leq N-1$. The finite family of asymptotically quasi-nonexpansive mappings $\left\{T_{n}\right\}_{n=0}^{N-1}$ is uniformly $L-$ Lipschitz, if

$\left\|T_{i}^{j} x-T_{i}^{j} y\right\| \leq L_{i, j}\|x-y\|, \forall x, y \in C$

for all $i \in\{0,1,2, \ldots, N-1\}, j \geq 1$, where $L \geq 1$.

Theorem 8. Let $\left\{T_{n}\right\}_{n=0}^{N-1}: C \rightarrow C$ be a finite uniformly L-Lipschitz family of asymptotically quasi-nonexpansive mappings with nonempty common fixed point set $F$. Assume that $\alpha_{n} \in(0,1]$, and $\beta_{n} \in[0,1]$ for all $n \in N$. Then $\left\{x_{n}\right\}$ generated by

$$
\left\{\begin{array}{lll}
x_{0} \in C=Q_{0}, & & \text { choosen arbitrarily, } \\
y_{n}=\left(1-\alpha_{n}\right) T_{i(n)}^{j(n)} x_{n}+\alpha_{n} T_{i(n)}^{j(n)} z_{n}, & & n \geq 0, \\
z_{n}=\left(1-\beta_{n}\right)+\beta_{n} T_{i(n)}^{j(n)} x_{n}, & n \geq 0, \\
C_{n}=\left\{z \in C:\left\|y_{n}-z\right\| \leq k_{i(n), j(n)}\right. & & \\
& \left.\left(1+\left(k_{i(n), j(n)}-1\right) \alpha_{n} \beta\right)\left\|x_{n}-z\right\|\right\} \cap A, & n \geq 0 \\
Q_{n}=\left\{z \in Q_{n-1}:\left\langle x_{n}-z, x_{0}-x_{n}\right\rangle \geq 0\right\}, & & n \geq 1 \\
& x_{n+1}=P_{\overline{c o} C_{n} \cap Q_{n}} x_{0}, &
\end{array}\right.
$$

converges strongly to $P_{F} x_{0}$.

We can drive the prove from the following two conclusions.

Conclusion 9. $\left\{T_{n=0}^{N-1}\right\}_{n=0}^{\infty}$ is a uniformly closed asymptotically family of countable quasi- $L_{n}$-Lipschitz mappings from $C$ into itself.

Conclusion 10. $F=\bigcap_{n=0}^{N} F\left(T_{n}\right)=\bigcap_{n=0}^{\infty} F\left(T_{i(n)}^{j(n)}\right)$, where $F(T)$ denotes the fixed point set of the mappings $T$.

Corollary 11. Let $T: C \rightarrow C$ be a L-Lipschitz asymptotically quasi-nonexpansive mappings with nonempty common fixed point set $F$. Assume that $\alpha_{n} \in(0,1]$, and $\beta_{n} \in[0,1]$ for all $n \in N$. Then $\left\{x_{n}\right\}$ generated by

$$
\begin{cases}x_{0} \in C=Q_{0}, & \text { choosen arbitrarily, } \\ y_{n}=\left(1-\alpha_{n}\right) T^{n} x_{n}+\alpha_{n} T^{n} z_{n}, & n \geq 0, \\ z_{n}=\left(1-\beta_{n}\right)+\beta_{n} T^{n} x_{n}, & n \geq 0 \\ C_{n}=\left\{z \in C:\left\|y_{n}-z\right\| \leq k_{n}\left(1+\left(k_{n}-1\right) \alpha_{n} \beta\right)\left\|x_{n}-z\right\|\right\} \cap A, & n \geq 0 \\ Q_{n}=\left\{z \in Q_{n-1}:\left\langle x_{n}-z, x_{0}-x_{n}\right\rangle \geq 0\right\}, & n \geq 1 \\ x_{n+1}=P_{\overline{c o} C_{n} \cap Q_{n}} x_{0}, & \end{cases}
$$

converges strongly to $P_{F} x_{0}$.

Proof. Take $T_{n} \equiv T$ in Theorem 8 , we get the desired result.

Author Contributions: All authors contributed equally to the writing of this paper. All authors read and approved the final manuscript.

Conflicts of Interest: "The authors declare no conflict of interest."

\section{References}

[1] Youla, D. C. (1987). Mathematical theory of image restoration by the method of convex projections. Image recovery: theory and application, 29-77.

[2] Tian, Z., Zarepisheh, M., Jia, X., \& Jiang, S. B. (2013). The fixed-point iteration method for IMRT optimization with truncated dose deposition coefficient matrix. arXiv preprint arXiv:1303.3504.

[3] Mann, W. R. (1953). Mean value methods in iteration. Proceedings of the American Mathematical Society, 4(3), 506-510. 
[4] Genel, A., \& Lindenstrauss, J. (1975). An example concerning fixed points. Israel Journal of Mathematics, 22(1), 81-86.

[5] Bauschke, H. H., \& Combettes, P. L. (2001). A weak-to-strong convergence principle for Fejér-monotone methods in Hilbert spaces. Mathematics of operations research, 26(2), 248-264.

[6] Matsushita, S. Y., \& Takahashi, W. (2005). A strong convergence theorem for relatively nonexpansive mappings in a Banach space. Journal of Approximation Theory, 134(2), 257-266.

[7] Guan, J., Tang, Y., Ma, P., Xu, Y., \& Su, Y. (2015). Non-convex hybrid algorithm for a family of countable quasi-Lipschitz mappings and application. Fixed Point Theory and Applications, 2015(1), 214.

[8] Nakajo, K., \& Takahashi, W. (2003). Strong convergence theorems for nonexpansive mappings and nonexpansive semigroups. Journal of Mathematical Analysis and Applications, 279(2), 372-379.

[9] Kim, T. H., \& Xu, H. K. (2006). Strong convergence of modified Mann iterations for asymptotically nonexpansive mappings and semigroups. Nonlinear Analysis: Theory, Methods E Applications, 64(5), 1140-1152.

[10] Martinez-Yanes, C., \& Xu, H. K. (2006). Strong convergence of the CQ method for fixed point iteration processes. Nonlinear Analysis: Theory, Methods E Applications, 64(11), 2400-2411.

[11] Su, Y., \& Qin, X. (2008). Monotone CQ iteration processes for nonexpansive semigroups and maximal monotone operators. Nonlinear Analysis: Theory, Methods \& Applications, 68(12), 3657-3664.

[12] Liu, Y., Zheng, L., Wang, P., \& Zhou, H. (2015). Three kinds of new hybrid projection methods for a finite family of quasi-asymptotically pseudocontractive mappings in Hilbert spaces. Fixed Point Theory and Applications, $2015(1), 118$. 